\title{
The Paradox of Virtual Dipoles in the Einstein Action
}

\author{
Giovanni Modanese* \\ California Institute for Physics and Astrophysics \\ 366 Cambridge Ave., Palo Alto, CA 94306 \\ and \\ University of Bolzano - Industrial Engineering \\ Via Sorrento 20, 39100 Bolzano, Italy
}

\begin{abstract}
The functional integral of pure Einstein $4 \mathrm{D}$ quantum gravity admits abnormally large and long-lasting "dipolar fluctuations", generated by virtual sources with the property $\int d^{4} x \sqrt{g(x)} \operatorname{Tr} T(x)=0$. These fluctuations would exist also at macroscopic scales, with paradoxical consequences. We set out their general features and give numerical estimates of possible suppression processes.
\end{abstract}

04.20.-q Classical general relativity.

04.60.-m Quantum gravity.

There has been considerable interest, in the last years, for the subject of vacuum fluctuations in quantum gravity. Several authors studied the possible occurrence of large fluctuations in $2+1$ dimensions, in different contexts [何. Other authors speculated whether the Planck scale fluctuations typical of quantum gravity, the so-called "spacetime foam", generate a noise which could be observed in certain circumstances [2]. General phenomenological models for the effects of the spacetime foam on high-energy scattering and dispersion relations were also proposed [3], based on the idea that the amplitudes of these effects might be suppressed just by a $M_{P}^{-1}$ factor and not by $M_{P}^{-2}$.

In this work we describe a set of gravitational field configurations, called "dipolar zero modes", which were not considered earlier in the literature. They give an exactly null contribution to the Einstein action, being thus candidates to become large fluctuations in the quantized theory. We give an explicit expression, to leading order in $G$, for some of the field configurations of this (actually quite large) set. We also give an estimate of possible suppression effects following the addition to the pure Einstein action of cosmological or $R^{2}$ terms. This letter is based upon the paper 近, which in turn settles and refines some partial previous work [5].

These zero modes have two peculiar features, which make them relatively easy to compute: (i) they are solutions of the Einstein equations, though with unphysical sources; (ii) their typical length scale is such that they can be treated in the weak field approximation. We shall see that these fluctuations can be large even on a "macroscopic" scale. There are some, for instance, which last $\sim 1 s$ or more and correspond to the field generated by a virtual source with size 
$\sim 1 \mathrm{~cm}$ and mass $\sim 10^{6} \mathrm{~g}$. This seems paradoxical, for several reasons, both theoretical and phenomenological. We have therefore been looking for possible suppression mechanisms. Our conclusion is that a vacuum energy term $(\Lambda / 8 \pi G) \int d^{4} x \sqrt{g(x)}$ in the action could do the job, provided it was scale-dependent and larger, at laboratory scale, than its observed cosmological value. This is at present only a speculative hypothesis, however.

The dipolar fluctuations owe their existence to the fact that the pure Einstein lagrangian $(1 / 8 \pi G) \sqrt{g(x)} R(x)$ has indefinite sign also for static fields. It is well known that the nonpositivity of the Einstein action makes an Euclidean formulation of quantum gravity difficult; in that context, however, the "dangerous" field configurations have small scale variations and could be eliminated, for instance, by some UV cut-off. This is not the case of the dipolar zero modes. They exist at any scale and do not make the Euclidean action unbounded from below, but have instead null (or $\ll \hbar$ ) action.

We shall consider the functional integral of pure quantum gravity, which represents a sum over all possible field configurations weighed with the factor $\exp \left[i \hbar S_{\text {Einstein }}\right]$ and possibly with a factor due to the integration measure. The Minkowski space is a stationary point of the vacuum action and has maximum probability. "Off-shell" configurations, which are not solutions of the vacuum Einstein equations, are admitted in the functional integration but are strongly suppressed by the oscillations of the exponential factor.

Due to the presence of the dimensional constant $G$ in the Einstein action, the most probable quantum fluctuations of the gravitational field "grow" at very short distances, of the order of $L_{\text {Planck }}=\sqrt{G \hbar / c^{3}} \sim 10^{-33} \mathrm{~cm}$. This led Wheeler, Hawking, Coleman and others to depict spacetime at the Planck scale as a "quantum foam" [6], with high curvature and variable topology. For a simple estimate (disregarding of course the possibility of topology changes, virtual black holes nucleation etc.), suppose we start with a flat configuration, and then a curvature fluctuation appears in a region of size $d$. How much can the fluctuation grow before it is suppressed by the oscillating factor $\exp [i S]$ ? (We set $\hbar=1$ and $c=1$ in the following.) The contribution of the fluctuation to the action is of order $R d^{4} / G$; both for positive and for negative $R$, the fluctuation is suppressed when this contribution exceeds $\sim 1$ in absolute value, therefore $|R|$ cannot exceed $\sim G / d^{4}$. This means that the fluctuations of $R$ are stronger at short distances - down to $L_{\text {Planck }}$, the minimum physical distance.

There is another way, however, to obtain vacuum field configurations with action smaller than 1 in natural units. Consider the Einstein equations and their covariant trace

$$
\begin{aligned}
& R_{\mu \nu}(x)-\frac{1}{2} g_{\mu \nu}(x) R(x)=-8 \pi G T_{\mu \nu}(x) ; \\
& R(x)=8 \pi G \operatorname{Tr} T(x)=8 \pi G g^{\mu \nu}(x) T_{\mu \nu}(x) .
\end{aligned}
$$

Then consider a solution $g_{\mu \nu}(x)$ of equation (11) with a source $T_{\mu \nu}(x)$ obeying the additional integral condition

$$
\int d^{4} x \sqrt{g(x)} \operatorname{Tr} T(x)=0 .
$$

Taking into account eq. (2) we see that the Einstein action computed for this solution is zero. Condition (3) can be satisfied by energy-momentum tensors that are not identically zero, provided they have a balance of negative and positive signs, such that their total integral is zero. Of course, they do not represent any acceptable physical source, but the corresponding solutions of (1) exist nonetheless, and are zero modes of the action. We shall give two explicit examples of virtual sources: (i) a "mass dipole" consisting of two separated mass distributions with different signs; (ii) two concentric "+/- shells". In both cases there are some parameters of the source which can be varied: the total positive and negative masses $m_{ \pm}$, their distance, the spatial extension of the sources. 
Suppose we have a suitable source, with some free parameters, and we want to adjust them in such a way to generate a zero-mode $g_{\mu \nu}(x)$ for which $S_{\text {Einstein }}[g]=0$. We shall always consider static sources where only the component $T_{00}$ is non vanishing. The action of their field is

$$
S_{z e r o-m o d e}=-\int d^{4} x \sqrt{g(x)} g^{00}(x) T_{00}(x) .
$$

To first order in $G$, one easily finds in Feynman gauge

$$
h_{\mu \nu}(\mathbf{x})=2 G\left(2 \eta_{\mu 0} \eta_{\nu 0}-\eta_{\mu \nu} \eta_{00}\right) \int d^{3} y \frac{T^{00}(\mathbf{y})}{|\mathbf{x}-\mathbf{y}|} .
$$

It is straightforward to check that $\sqrt{g(x)} g^{00}(x)=1+o\left(G^{2}\right)$ and thus $S_{\text {zero-mode }}=$ $-\int d^{4} x T_{00}(x)+o\left(G^{2}\right)$. Therefore provided the integral of the mass-energy density vanishes, the action of our field configuration is of order $G^{2}$, i.e., practically negligible, as we shall see now with a numerical example. Let us choose the typical parameters of the source as follows:

$$
r \sim 1 \mathrm{~cm} ; \quad m_{ \pm} \sim 10^{k} \mathrm{~g} \simeq 10^{37+k} \mathrm{~cm}^{-1}
$$

(implying $r_{S c h w .} / r \sim 10^{-29+k}$ ). We assume in general an adiabatic switch-on/off of the source, thus the time integral contributes to the action a factor $\tau$. We shall keep $\tau$ (in natural units) very large, in order to preserve the static character of the field. Here, for instance, let us take $\tau \sim 1 \mathrm{~s} \simeq 3 \cdot 10^{10} \mathrm{~cm}$. With these parameters one finds

$$
S_{\text {zero-mode }}^{\text {order }} G^{2} \sim \tau \frac{G^{2} m_{ \pm}^{2}}{r^{3}} \sim 10^{-20+3 k}
$$

Thus the field generated by a virtual source with typical size (雨), satisfying the condition $\int d^{3} x T_{00}(\mathbf{x})=0$, has negligible action even with $k=6$ (corresponding to apparent matter fluctuations with a density of $10^{6} \mathrm{~g} / \mathrm{cm}^{3}$ !) This should be compared to the huge action of the field of a single, unbalanced virtual mass $m$; with the same values we have $S_{\text {single } m}=$ $-\int d^{4} x \sqrt{g(x)} \operatorname{Tr} T(x) \sim \tau m+o\left(G^{2}\right) \sim 10^{47+k}$.

This numerical estimate shows that the cancellation of the first order term in $S_{z e r o-m o d e}$ allows to obtain a simple lower bound on the strength of the fluctuations. In principle, however, one could always find all the terms in the classical weak field expansion, proportional to $G$, $G^{2}, G^{3}$, etc., and adjust $T_{00}$ as to have $S_{z e r o-m o d e}=0$ exactly. They can be represented by those Feynman diagrams of perturbative quantum gravity which contain vertices with $3,4 \ldots$ gravitons but do not contain any loops. The ratio between each contribution to $S$ and that of lower order in $G$ has typical magnitude $r_{S c h w .} / r$, where $r_{S c h w} .=2 \pi G m_{ \pm}$is the Schwarzschild radius corresponding to one of the two masses and $r$ is the typical size of the source. For a wide range of parameters, this ratio is very small, so the expansion converges quickly.

As a first example of unphysical source satisfying (3), consider the static field produced by a mass dipole. This consists of a positive source with mass $m_{+}$and radius $r_{+}$and a negative source with mass $-m_{-}$and radius $r_{-}$, placed a distance $2 a$ apart. The radii of the two sources are such that $a \gg r_{ \pm} \gg r_{S c h w}$, where $r_{S c h w}$. is the Schwarzschild radius corresponding to the mass $m_{+}$. The action is found to be $S_{\text {Dipole }}=-\int d^{4} x T_{00}(\mathbf{x})=-\tau\left(m_{+}-m_{-}\right)+o\left(G^{2}\right)$. This vanishes for $m_{+}=m_{-}$, apart from terms of order $G^{2}$ (i.e., our dipoles have in reality a tiny monopolar component). The values of the masses and the radii $r_{ \pm}$(both of order $r$ ) can vary in a continuous way - provided the condition above is satisfied. Therefore these (non singular) "dipolar" fields constitute a subset with nonzero volume in the functional integration. In fact, they are only a small subset of all solutions of the Einstein equations with sources satisfying eq. (3). 
Another example is given by two concentric spherical shells, the internal one with radii $r_{1}, r_{2}$, and the external one with radii $r_{2}, r_{3}\left(r_{1}<r_{2}<r_{3}\right)$. Let the internal shell have mass density $\rho_{1}$ and the external shell density $\rho_{2}$, with opposite sign. The condition for zero action requires, up to terms of order $G^{2}$, that the total positive mass equals the total negative mass, i.e., $\rho_{1}\left(r_{2}^{3}-r_{1}^{3}\right)+\rho_{2}\left(r_{3}^{3}-r_{2}^{3}\right)=0$. The spherical symmetry of this source offers some advantages in the calculations.

One may think that large gravitational fluctuations, if real, would not remain unnoticed. Even though vacuum fluctuations are homogeneous, isotropic and Lorentz-invariant, they could manifest themselves as noise of some kind. Most authors are skeptic about the possibility of detecting the noise due to spacetime foam [2, 3], but the virtual dipole fluctuations described in this paper are much closer to the laboratory scale. Observable quantities, like for instance the connection coefficients $\Gamma_{\mu \nu}^{\rho}$ could then exhibit strong fluctuations.

The existence of these fluctuations would be paradoxical, however, already at the purely conceptual level. Common wisdom in particle physics states that the vacuum fluctuations in free space correspond to virtual particles or intermediate states which live very short, i.e., whose lifetime is close to the minimum allowed by the Heisenberg indetermination relation.

Let us estimate the product $E \tau$ for the dipolar fluctuations. The total energy of a static gravitational field configuration vanishing at infinity is the ADM energy. Since the source of a dipolar fluctuation satisfies the condition $\int d^{3} x T_{00}(\mathbf{x})=0$ up to terms of order $G^{2}$, the dominant contribution to the ADM energy is the Newtonian binding energy [7].

The binding energy of the field generated by a source of mass $m$ and size $r$ is of the order of $E \sim-G m^{2} / r$, where the exact proportionality factor depends on the details of the mass distribution. For a dipolar field configuration characterized by masses $m_{+}$and $m_{-}$and radii of the sources $r_{+}$and $r_{-}$, the total gravitational energy is of the order of $E_{t o t} \sim-G m_{ \pm}^{2}\left(r_{-}^{-1}+r_{+}^{-1}\right)$ (disregarding the interaction energy between the two sources, proportional to $1 / a \ll 1 / r$ ). With the parameters (4) we have $E_{\text {tot }} \sim-G m_{ \pm}^{2} \sim-10^{12+k} \mathrm{~cm}^{-1}$. Remembering that $k$ can take values up to $k=6$, we find for these dipolar fluctuations $\tau\left|E_{t o t}\right| \sim 10^{28}$ ! (For comparison, remember the case of a "monopole" fluctuation of virtual mass $m$ and duration $\tau$. The condition $S<1$ implies $\tau m<1$. The dominant contribution to the ADM energy is just $m$, so the rule $E \tau<1$ is respected.)

The Newtonian binding energy of the concentric $+/$ - shells turns out to be of the same magnitude order, more exactly $E=\frac{G m_{ \pm}}{r} P(\beta)$, where $P(\beta) \equiv P\left(r_{3} / r_{2}\right)$ is a polynomial which is positive if $\left|\rho_{1}\right|>\left|\rho_{2}\right|$ (the repulsion between the two shells predominates) and negative if $\rho_{1}<\rho_{2}$ (the attraction inside each shell predominates). From the physical point of view it is reasonable to admit - remembering that we are in a weak field regime and forgetting general covariance for a minute - that the binding energy is localized within the surface of the outer shell (the field is $o\left(G^{2}\right)$ outside). The energy density is therefore of the order of $\frac{|E|}{r^{3}} \sim \frac{G m_{ \pm}}{r^{4}} \sim 10^{29+k} \mathrm{~cm}^{-4}$ (with the parameters (舟), and can take both signs. This value looks quite large, even though the Ford-Roman inequalities [8] or similar bounds do not apply to quantum gravity, where the metric is not fixed but free to fluctuate, and there is in general no way to define a local energy density.

Concerning possible suppression processes of the dipolar fluctuations, here we just quote the results. The contribution to the cosmological term is $\Delta S_{\Lambda} \sim \tau \Lambda m_{ \pm} r^{2} \sim 10^{-3+k}$, with the parameters above, and the contribution to an $\alpha R^{2}$ term is of the order of $\tau G^{2} m_{ \pm}^{2} / r^{3} \sim \alpha 10^{-48+2 k}$. We see that only the cosmological term can act as a cut-off at macroscopic scales.

Acknowledgment - This work was supported in part by the California Institute for Physics and Astrophysics via grant CIPA-MG7099. 
* e-mail address: giovanni.modanese@unibz.iz

\section{References}

[1] A. Ashtekar, Phys. Rev. Lett. 77, 4864 (1996). R. Gambini and J. Pullin, Mod. Phys. Lett. A 12, 2407 (1997). A. E. Dominguez and M. H. Tiglio, Phys. Rev. D 60, 064001 (1999).

[2] G. Amelino-Camelia, Nature 398, 216 (1999); Phys. Lett. B 477, 436 (2000). R. J. Adler, I. M. Nemenman, J. M. Overduin and D. I. Santiago, Phys. Lett. B 477, 424 (2000).

[3] A. A. Kirillov, Sov. Phys. JETP (J. Exp. Theor. Phys.) 88, 1051 (1999). J. Ellis, N. E. Mavromatos and D. V. Nanopoulos, Phys. Rev. D 61, 027503 (2000).

[4] G. Modanese, Large "Dipolar" Vacuum Fluctuations in Quantum Gravity (gr-qc/0005009).

[5] G. Modanese, Phys. Rev. D 59, 024004 (1998); Phys. Lett. B 460, 276 (1999).

[6] J.A. Wheeler, Ann. Phys. 2 (1957) 604. S.W. Hawking, Nucl. Phys. B 144, 349 (1978). S. Coleman, Nucl. Phys. B 310, 643 (1988).

[7] N.O. Murchadha and J.W. York, Phys. Rev. D 10 (1974) 2345.

[8] L.H. Ford and T.A. Roman, Phys. Rev. D 43, 3972 (1991); D 46, 1328 (1992); D 51, 4277 (1995); D 55, 2082 (1997). 